\title{
BMJ Open Absolute risk and risk factors for stroke mortality in patients with end-stage kidney disease (ESKD): population- based cohort study using data linkage
}

\author{
Nicole Louise De La Mata, ${ }_{1}^{1}$ Maria Alfaro-Ramirez, ${ }^{2}$ Patrick J Kelly, ${ }^{1}$ Philip Masson, ${ }^{3}$ \\ Rustam Al-Shahi Salman, ${ }^{4}$ Angela C Webster ${ }^{5}$
}

To cite: De La Mata NL, Alfaro-Ramirez M, Kelly PJ, et al. Absolute risk and risk factors for stroke mortality in patients with end-stage kidney disease (ESKD): populationbased cohort study using data linkage. BMJ Open 2019;9:e026263. doi:10.1136/ bmjopen-2018-026263

- Prepublication history and additional material for this paper are available online. To view these files, please visit the journal online (http://dx.doi org/10.1136/bmjopen-2018026263).

Received 25 August 2018 Revised 6 November 2018 Accepted 19 December 2018

\section{Check for updates}

(c) Author(s) (or their employer(s)) 2019. Re-use permitted under CC BY-NC. No commercial re-use. See rights and permissions. Published by BMJ.

${ }^{1}$ Sydney School of Public Health, University of Sydney, Sydney, New South Wales, Australia ${ }^{2}$ School of Mathematics and Applied Statistics, University of Wollongong, Wollongong, New South Wales, Australia ${ }^{3}$ Department of Renal Medicine, Royal Free London NHS Foundation Trust, London, UK ${ }^{4}$ Centre for Clinical Brain Sciences, University of Edinburgh, Edinburgh, UK ${ }^{5}$ School of Public Health, The University of Sydney, Sydney, New South Wales, Australia

Correspondence to Dr Nicole Louise De La Mata; nicole.delamata@sydney.edu.au

\section{ABSTRACT}

Introduction People with end-stage kidney disease (ESKD) have up to 30-fold higher risk of stroke than the general population.

Objective To determine risk factors associated with stroke death in the ESKD population.

Methods We identified all patients with incident ESKD in Australia (1980-2013) and New Zealand (1988-2012) from the Australian and New Zealand Dialysis and Transplant Registry (ANZDATA) registry. We ascertained underlying cause of death from data linkage with national death registries and risk factors from ANZDATA. Using a competing risks multivariable regression model, we estimated cumulative incidence of stroke and non-stroke deaths, and risk factors for stroke deaths (adjusted sub$H R, S H R)$.

Results We included 60823 people with ESKD. There were 941 stroke deaths and 33377 non-stroke deaths during 381874 person-years of follow-up. Overall, the cumulative incidence of stroke death was $0.9 \%$ and non-stroke death was 36.8\% 5 years after starting ESKD treatment. The risk of stroke death was higher at older ages (SHR 1.92, 95\% Cl 1.45 to 2.55), in females (SHR $1.41,95 \% \mathrm{Cl} 1.21$ to 1.64$)$, in people with cerebrovascular disease (SHR 2.39, 95\% Cl 1.99 to 2.87), with ESKD caused by hypertensive/renovascular disease (SHR 1.39, $95 \% \mathrm{Cl} 1.09$ to 1.78 ) or polycystic kidney disease (SHR $1.38,95 \% \mathrm{Cl} 1.00$ to 1.90 ), with earlier year of ESKD treatment initiation (SHR 1.93, 95\% Cl 1.56 to 2.39) and receiving dialysis (transplant vs haemodialysis SHR 0.27 , $95 \% \mathrm{Cl} 0.09$ to 0.84 ).

Conclusion Patients with ESKD with higher risk of stroke death are older, women, with cerebrovascular disease, with hypertensive/renovascular or polycystic kidney disease cause of ESKD, with earlier year of ESKD treatment and receiving dialysis. These groups may benefit from targeted stroke prevention interventions.

\section{INTRODUCTION}

Compared with the general population, people with end-stage kidney disease (ESKD) have a substantially reduced life expectancy with cardiovascular disease killing more than $50 \%$ of people receiving renal replacement
Strengths and limitations of this study

- This study used a large, population-based cohort including the entire population with end-stage kidney disease (ESKD) in Australia and New Zealand.

- The largest multinational study examining risk factors for stroke mortality in people with ESKD to date.

- Data linkage with the national death registries allowed legal cause of death to be ascertained for all patients, although probabilistic data linkage for Australian patients with ESKD may lead to some patients being incorrectly linked.

- Detailed patient information on the use of stroke prevention drugs or other factors relating to their risk of stroke was not available, such as family history of stroke and atrial fibrillation.

therapy (RRT).${ }^{1-3}$ Globally in developed countries, mortality from cardiovascular diseases has declined since the $1960 \mathrm{~s}$, with heart disease deaths declining by $56 \%$ in the USA between 1950 and 1996 and all-cause cardiovascular deaths declining by more than $70 \%$ in the Netherlands and $60 \%$ in the UK and Ireland from 1980 to 2009. Despite people with ESKD having a 5 to 30 -fold increased risk of stroke compared with the general population, the epidemiology of stroke death in ESKD is far less clear. An Australian registry-based study reported that for people with ESKD, the relative risk of death from cardiovascular disease between 1992 and 2005 increased compared with the general population, despite absolute cardiovascular death rates falling. ${ }^{4}$ Another contemporary Taiwanese case-control study reported a greater than twofold risk of 30-day mortality following a stroke in people with ESKD compared with propensity-matched controls. $^{5}$

Such differences in outcomes between the general population and people with ESKD following a stroke could arise because of 
differences in the prevalence and severity of underlying traditional stroke risk factors including hypertension, diabetes and atrial fibrillation. ${ }^{67}$ Stroke case severity may also differ, as might the effectiveness and access to preventative treatments (including aspirin, ACE inhibitors, anticoagulants and statins) and acute stroke interventions including thrombolysis. Risk factors specific to people with ESKD may also exist: accelerated calcific atherosclerosis (from disordered bone metabolism), platelet dysfunction, anaemia. ${ }^{18}$ In addition, dialysis-associated osmotic fluid and electrolyte shifts or exposure to glucose degradation products from peritoneal dialysis (PD) fluid may all alter normal pathological glial scar resolution and affect functional outcomes following stroke. ${ }^{9}$ For people with kidney transplants, in addition to traditional cardiovascular risk factors, graft function and albuminuria as well as the side effects of immunosuppressive medicines might all modify stroke outcomes. ${ }^{10}$

The aims of our study were to establish robust estimates of stroke and non-stroke death incidence in people with ESKD, and to identify risk factors associated with dying after a stroke.

\section{METHOD}

\section{Study design and setting}

We performed a population-based cohort study of all adults and children starting RRT for treatment of ESKD in Australia (1 January 1980 to 31 December 2013) and New Zealand (1 January 1988 to 31 December 2012). Australia (population 24.8 million in 2017) and New Zealand (population 4.7 million in 2017) are broadly comparable in racial make-up and population demographics, where approximately $75 \%$ are from a European, Australian or New Zealand cultural background. ${ }^{1112}$ Both countries have a universal healthcare system that provides free medical care in public health systems. All deaths occurring in each country are reported to the Births, Deaths and Marriages Registry with a medical certificate for the cause of death completed by a medical doctor which is then coded to the international standard, the International Statistical Classification of Diseases and Related Health Problems (ICD), codes by a qualified clinical coder, detailing both the underlying and secondary causes of death. The underlying cause of death is considered as the disease or condition which initiated the sequence of events resulting in death. Secondary or contributing causes of death are other diseases or conditions that contributed to the death but were not the underlying cause.

\section{Participants, data linkage and death outcomes \\ People with ESKD}

The Australia and New Zealand Dialysis and Transplant Registry (ANZDATA) prospectively collects data from all dialysis and transplant centres in Australia and New Zealand. Data are collected either via web based or printed survey forms in real time as key events occur for each patient with ESKD. Core data collected include demographics (age, sex, country, racial background, body mass index, BMI and smoking history), comorbidities (cerebrovascular disease, diabetes, coronary artery disease, peripheral artery disease and previous malignancy) and modality of treatment for ESKD (type of dialysis or transplant, date initiated and cause of kidney failure). ANZDATA data collection, remit and validation has been previously described elsewhere. ${ }^{13}$

\section{Data linkage}

We used data linkage of ANZDATA with each of the national death registries in Australia and New Zealand to determine the date and underlying cause of death for people with ESKD. In Australia, patients with ESKD were linked with the National Death Index, which records all deaths from 1980 and is maintained by the Australian Institute of Health and Welfare (AIHW). The Australian Bureau of Statistics (ABS) retrieves information on all deaths and their causes from the Births, Deaths and Marriages Registry in each state and territory. The ABS collates these data and a clinical coder then codes the causes of death to an international standard ICD. ${ }^{14}$ The National Centre for Classification in Health has developed an Australian modification version, currently in its Tenth Revision which is referred to as ICD-10-AM. We used probabilistic record linkage for Australian patients with ESKD, matching on date of birth, sex and full name. In New Zealand, patients with ESKD were linked with the Mortality Collection database, which collects information on all deaths from 1988 and is maintained by the New Zealand Ministry of Health. The Ministry receives information from the Births, Deaths and Marriages Registry as well as additional information from medical certificates and coroners' reports to classify causes of death into ICD-10-AM. We used deterministic linkage for New Zealand patients with ESKD, matching on the National Health Index number. Hence, our analysis was restricted by the available data in the national death registries. We included all patients with incident ESKD in Australia during 1980-2013 and in New Zealand during 1988-2012.

Data linkage was performed by the AIHW and New Zealand Ministry of Health using best-practice privacy-preserving protocols. After data linkage was complete, only de-identified data were made available for the purposes of this study.

\section{Death ascertainment, stroke definition and cause of death}

Causes of death were determined using ICD-10-AM diagnosis codes provided in the underlying cause of death from the Australian and New Zealand national death registries. All-cause stroke deaths included subarachnoid haemorrhage (I60.0-I60.9), intracerebral haemorrhages (I61.0-I61.9), intracranial haemorrhages (I62.0-I62.9), ischaemic strokes (I63.0-I63.9), unspecified strokes (I64.0-I64.9) and, transient cerebral ischaemic attacks and related syndromes (G45.0-G45.9). Prior to 1997, the ninth revision of ICD was implemented. There is little evidence for significant differences in stroke coding 


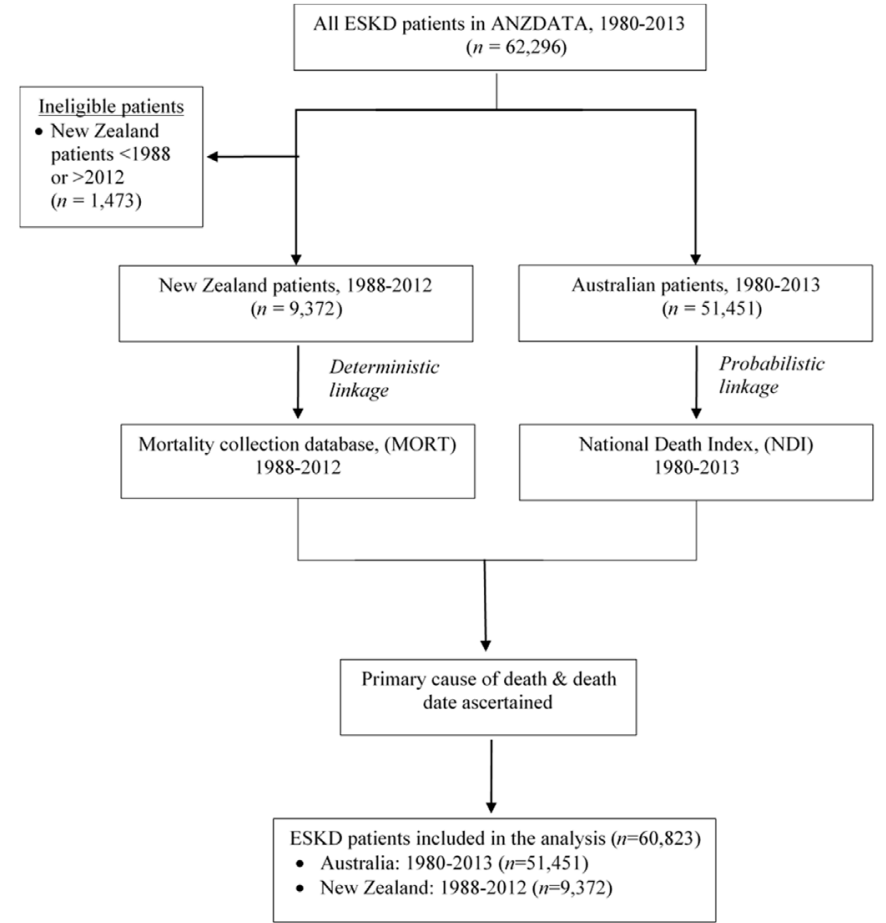

Figure 1 Patient flow chart of data linkage process and inclusion into analysis. ANZDATA, Australian and New Zealand Dialysis and Transplant Registry; ESKD, end-stage kidney disease.

between revisions, with agreement being over $90 \% .^{15} 16$ All other causes of death were considered as non-stroke deaths.

\section{Patient and public involvement}

We used retrospective registry data for this study, hence study participants were not involved in the recruitment or design of the study. Patient advisers were also not involved in this study.

\section{Statistical analyses}

We summarised the main causes of death by deriving the leading causes of death, defined by ICD-10-AM codes. ${ }^{17}$ We also estimated the crude mortality rates for stroke and non-stroke deaths, overall and stratified by patient demographics.

We used Fine and Gray competing risks regression models to evaluate risk factors associated with stroke death. ${ }^{18}$ Time was measured from the start date of ESKD treatment until a participant died or 31 December 2013 (Australian participants) or 31 December 2012 (New Zealand participants), whichever came first. As probabilistic record linkage may lead to a small proportion of incorrect links, ${ }^{19}$ Australian patients were censored at the ANZDATA date of death if ANZDATA considered the patient had died and the national death registry had not captured any death. For the analysis of stroke deaths, all non-stroke deaths were considered as competing events. For comparison, we also similarly conducted an analysis of non-stroke deaths, with stroke deaths considered as competing events. For both the stroke and non-stroke analyses, we used the same covariates, selected a priori including: age at initiation of ESKD treatment, sex, year of initiating ESKD treatment, country, racial background, BMI category (underweight, $\leq 18.4 \mathrm{~kg} / \mathrm{m}^{2}$; normal, $18.5-$ $24.9 \mathrm{~kg} / \mathrm{m}^{2}$; overweight, $25.0-29.9 \mathrm{~kg} / \mathrm{m}^{2}$ and obese, $\geq 30.0 \mathrm{~kg} / \mathrm{m}^{2}$ ), initial ESKD treatment modality, comorbidities, smoking status and cause of ESKD. Univariable models were fitted for each covariate, and the multivariable models included all covariates. The cumulative incidence was estimated from the final multivariate model, by sex and year of initiating ESKD treatment, adjusting for all other covariates except BMI.

There were no missing data, except for BMI. Missing values for BMI were imputed using chained equations with five iterations. To impute, we used the same variables as those used in the competing risks analyses, then converted those BMI values into categories for the regression models.

We conducted a further three sensitivity analyses to determine: (1) whether risk factors for any stroke death were similar to risk factors for fatal ischaemic stroke and fatal haemorrhagic stroke; (2) whether risk factors were the same when stroke death was defined as underlying cause, or secondary cause when kidney failure was indicated as the underlying cause and (3) whether ever transplanted with a kidney as a time-varying covariate produced different model estimates. For these sensitivity analyses, we used cause-specific Cox regression models to evaluate associated risk factors and used the same covariates and definition of time at risk as described in the main analysis.

Data were analysed using Stata V.14 (Stata).

\section{RESULTS}

\section{Patient characteristics}

We identified a total of 62296 people with incident ESKD in Australia and New Zealand between 1980 and 2013. Of these, 1473 New Zealand patients initiated RRT prior to 1988 or after 2012, and so could not be linked. The remaining 60823 patients with ESKD were included in the analysis (figure 1). The fact of death for the majority of patients was similar between ANZDATA and the national death registries, where disagreement occurred for $7.6 \%$ of Australian patients and $5.5 \%$ of New Zealand patients. There were 467 patients considered to be lost to follow-up in ANZDATA, where 57 patients were considered to have died in the national death registry. There were 381874 person-years (PYs) of follow-up, with a median follow-up time of 4.0 years (IQR 1.7-8.4 years).

During the study period, 26505 patients (43\%) remained in active follow-up and 34318 patients (57\%) died (table 1). Of the patients who died, 33377 patients died of non-stroke causes $(97.3 \%)$ and 941 patients $(2.7 \%)$ died of stroke. In our entire study population, two-thirds $(69 \%)$ were aged 50 years or older at ESKD, with a median age of 59 years (IQR 46-69). Most were from Australia (85\%), were male (59\%), had a Caucasian background $(76 \%)$ and had started treatment for ESKD 
Table 1 Characteristics of participants with end-stage kidney disease (ESKD), overall and by stroke and non-stroke deaths

\begin{tabular}{|c|c|c|c|}
\hline & Stroke deaths Total & $\begin{array}{l}\text { Non-stroke deaths } \\
\text { Total }\end{array}$ & Total \\
\hline Characteristics & n (\%) ${ }^{*}$ & n (\%)* & n (\%) ${ }^{*}$ \\
\hline Total, (\%) & $941(2) \dagger$ & $33377(55) \dagger$ & $60823(100)$ \\
\hline \multicolumn{4}{|l|}{ Age at RRT initiation (years) } \\
\hline$\leq 29$ & $25(3)$ & $1150(3)$ & $5088(8)$ \\
\hline $30-49$ & 177 (19) & $5399(16)$ & $13807(23)$ \\
\hline $50-64$ & $329(35)$ & 11417 (34) & $19980(33)$ \\
\hline $65-74$ & $261(28)$ & $9658(29)$ & $13842(23)$ \\
\hline$\geq 75$ & $149(16)$ & $5753(17)$ & 8106 (13) \\
\hline Median (IQR) & $62(52-71)$ & 63 (53-72) & $59(46-69)$ \\
\hline \multicolumn{4}{|l|}{ Sex } \\
\hline Female & $472(50)$ & $13901(42)$ & $25042(41)$ \\
\hline Male & $469(50)$ & $19476(58)$ & $35781(59)$ \\
\hline \multicolumn{4}{|l|}{ Year of ESKD } \\
\hline$\leq 1995$ & $379(40)$ & $11515(35)$ & $15382(25)$ \\
\hline 1996-2005 & 367 (39) & $14700(44)$ & $22115(36)$ \\
\hline 2006-2013 & $195(21)$ & $7162(21)$ & $23326(38)$ \\
\hline \multicolumn{4}{|l|}{ Country } \\
\hline Australia & $819(87)$ & $28173(84)$ & $51451(85)$ \\
\hline New Zealand & $122(13)$ & $5204(16)$ & $9372(15)$ \\
\hline \multicolumn{4}{|l|}{ Racial background } \\
\hline European & $737(78)$ & $26286(79)$ & $46025(76)$ \\
\hline Indigenous Oceania & $122(13)$ & $5244(16)$ & 9743 (16) \\
\hline Asian & $78(8)$ & $1667(5)$ & $4357(7)$ \\
\hline African and Middle East & $4(<0.5)$ & $154(<0.5)$ & $492(1)$ \\
\hline Peoples of the America & $0(-)$ & $4(<0.1)$ & $19(<0.1)$ \\
\hline Other $\neq$ & $0(-)$ & $22(<0.1)$ & $187(<0.4)$ \\
\hline \multicolumn{4}{|l|}{ Body mass index } \\
\hline Underweight ( $\leq 18.4)$ & $32(3)$ & $1274(4)$ & $2702(4)$ \\
\hline Normal (18.5-24.9) & $353(38)$ & $10662(32)$ & $19866(33)$ \\
\hline Overweight (25.0-29.9) & $209(22)$ & $8670(26)$ & $16641(27)$ \\
\hline Obese $(\geq 30)$ & $132(14)$ & $6561(20)$ & $13772(23)$ \\
\hline Not collected & $215(23)$ & $6210(19)$ & 7842 (13) \\
\hline \multicolumn{4}{|l|}{ Comorbidities at ESKD } \\
\hline Cerebrovascular disease & $246(26)$ & $5393(16)$ & $7676(13)$ \\
\hline Diabetes & $313(33)$ & $12863(39)$ & $21717(36)$ \\
\hline Coronary artery disease & $372(40)$ & $14310(43)$ & 20785 (34) \\
\hline Peripheral artery disease & $252(27)$ & $9487(28)$ & $13373(22)$ \\
\hline Previous malignancy & $208(22)$ & 9707 (29) & $15788(26)$ \\
\hline \multicolumn{4}{|l|}{ Smoking status } \\
\hline Current/former & $413(44)$ & $16081(48)$ & $28389(47)$ \\
\hline Never & $345(37)$ & $11955(36)$ & $25861(43)$ \\
\hline Unknown & $183(19)$ & $5341(16)$ & $6573(11)$ \\
\hline \multicolumn{4}{|l|}{ Cause of renal failure } \\
\hline Diabetes & $249(26)$ & 9925 (30) & $16909(28)$ \\
\hline
\end{tabular}




\begin{tabular}{|c|c|c|c|}
\hline & Stroke deaths Total & $\begin{array}{l}\text { Non-stroke deaths } \\
\text { Total }\end{array}$ & Total \\
\hline Characteristics & n (\%)* & n (\%)* & n $(\%)^{*}$ \\
\hline $\begin{array}{l}\text { Glomerulonephritis/lgA } \\
\text { nephropathy }\end{array}$ & $211(22)$ & $7626(23)$ & $16632(27)$ \\
\hline Other & $241(26)$ & $9440(28)$ & $16015(26)$ \\
\hline $\begin{array}{l}\text { Transplanted at beginning of } \\
\text { study }\end{array}$ & $4(<0.5)$ & $151(<0.5)$ & $1649(3)$ \\
\hline Transplanted during study & $154(16)$ & 4978 (15) & $16068(26)$ \\
\hline
\end{tabular}

${ }^{*}$ Column percentage.

†Row percentage.

łIncludes Australian Aboriginal, Maori, Torres Strait and Pacific Islander.

$\mathrm{RRT}$, renal replacement therapy.

in 1996 or later $(74 \%)$. The majority of patients had a normal $(33 \%)$ or overweight (27\%) BMI, where only $4 \%$ were underweight. The BMI was not collected for $13 \%$ of patients, the large majority (95\%) of whom reached ESKD prior to 1996. The main cause of ESKD was diabetes (28\%), followed by glomerulonephritis/IgA nephropathy (27\%), other causes of ESKD (26\%), hypertension/renal artery disease (12\%) and polycystic kidney disease $(7 \%)$. The reason for other causes of ESKD included uncertain diagnosis $(23 \%)$, analgesic nephropathy $(18 \%)$ and reflux nephropathy $(16 \%)$.

\section{Deaths}

There were a total of 34318 deaths since initiation of ESKD treatment, with a crude rate of 89.9 (95\% CI 88.9 to 90.8 ) per 1000 PYs. The top four leading causes of death were recorded as diabetes $(18 \%)$, coronary heart disease $(18 \%)$, kidney failure $(13 \%)$ and glomerular disease $(5 \%)$. Stroke was the sixth leading cause of death since RRT, with a total 941 stroke deaths, corresponding to a crude rate of 2.5 (95\% CI 2.3 to 2.6) per 1000 PYs (table 2). Stroke deaths were due to: 108 (11\%) ischaemic strokes; 259 (28\%) intracerebral haemorrhages; 90 $(10 \%)$ intracranial haemorrhages; 68 (7\%) subarachnoid haemorrhages; 114 (12\%) transient cerebral ischaemic attacks and related syndromes and 302 (32\%) unspecified strokes (online supplementary appendix 1 ).

The highest rate of stroke death since initiation of ESKD treatment was among those with known cerebrovascular disease at 9.1 (95\% CI 8.0 to 10.3$)$ per 1000 PYs, followed by those aged 75 years or older at 6.6 (95\% CI 5.6 to 7.7 ) per 1000 PYs (table 2). In comparison, the highest rate of non-stroke death since initiation of ESKD treatment was among those aged 75 years or older at 254.5 (95\% CI 248.0 to 261.2) per 1000 PYs, followed by those with cerebrovascular disease at 199.0 (95\% CI 193.7 to 204.4) per 1000 PYs.

\section{Risk factors for stroke mortality}

The multivariable model suggested a higher risk of stroke death was associated with older age, female sex, earlier year of ESKD, normal BMI range, presence of pre-existing cerebrovascular disease, no previous malignancy, ESKD treatment with haemodialysis (HD) compared with transplant and hypertension/renal artery disease or polycystic kidney disease as the cause of ESKD compared with glomerulonephritis/IgA nephropathy (table 3).

Older age was associated with a higher risk of stroke death, where patients aged 75 years or older were $92 \%$ sub-HR (SHR 1.92, 95\% CI 1.45 to 2.55 ) more likely to die from stroke death compared with those aged 30-49 years $(p<0.001)$. Females were $41 \%$ more likely to die of stroke death compared with males (SHR 1.41, 95\% CI 1.21 to 1.64; $\mathrm{p}<0.001)$. There was a decreasing trend in the risk of stroke death overtime, where patients initiating ESKD treatment in $\leq 1995$ and 1996-2005 were 93\% (SHR 1.93, $95 \%$ CI 1.56 to 2.39 ) and $30 \%$ (SHR $1.30,95 \%$ CI 1.09 to $1.55)$, respectively, more likely to die from stroke death compared with those in 2006-2013 ( $\mathrm{p}<0.001)$. Patients had over two times the increased risk of stroke death if they had pre-existing cerebrovascular disease (SHR 2.39, $95 \%$ CI 1.99 to $2.87 ; \mathrm{p}<0.001$ ) and were $36 \%$ less likely to die of stroke if they had a previous malignancy (SHR 0.64, $95 \%$ CI 0.53 to $0.78 ; \mathrm{p}<0.001)$. There was no difference in the risk of stroke death for those who initiated PD (SHR 0.85 ; $95 \%$ CI 0.72 to 1.01 ) compared with HD. However, transplant patients were $73 \%$ less likely to die of stroke compared with HD patients (SHR 0.27, 95\% CI 0.09 to $0.84)$. Similar estimates for the risk of stroke death were produced when transplant was considered a time-varying covariate (HR 0.24, 95\% CI 0.17 to $0.32 ; \mathrm{p}<0.001$ ) (online supplementary appendix 2). The cause of ESKD was also significantly associated with stroke mortality $(\mathrm{p}=0.020)$. Patients with hypertension/renal artery disease or 


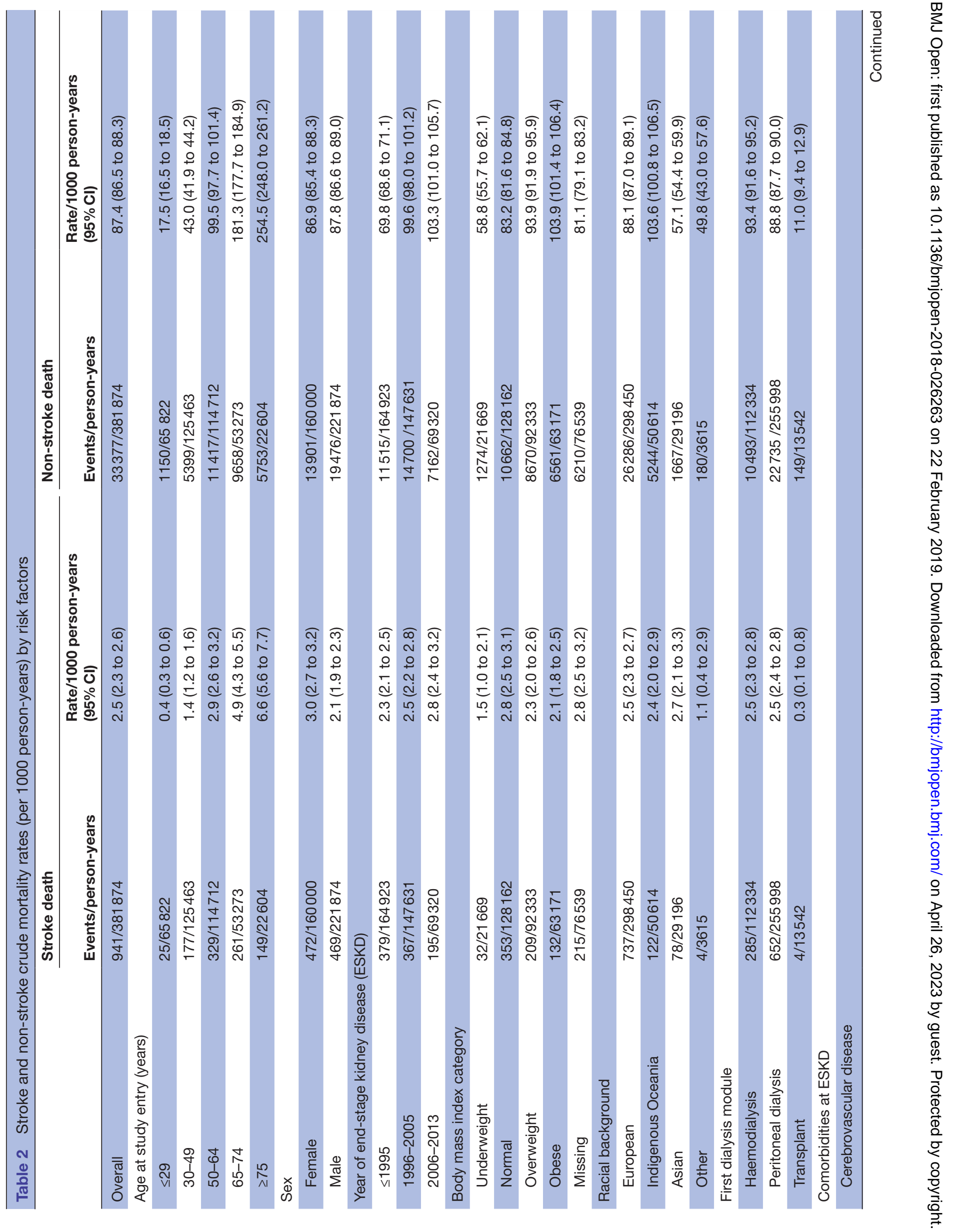




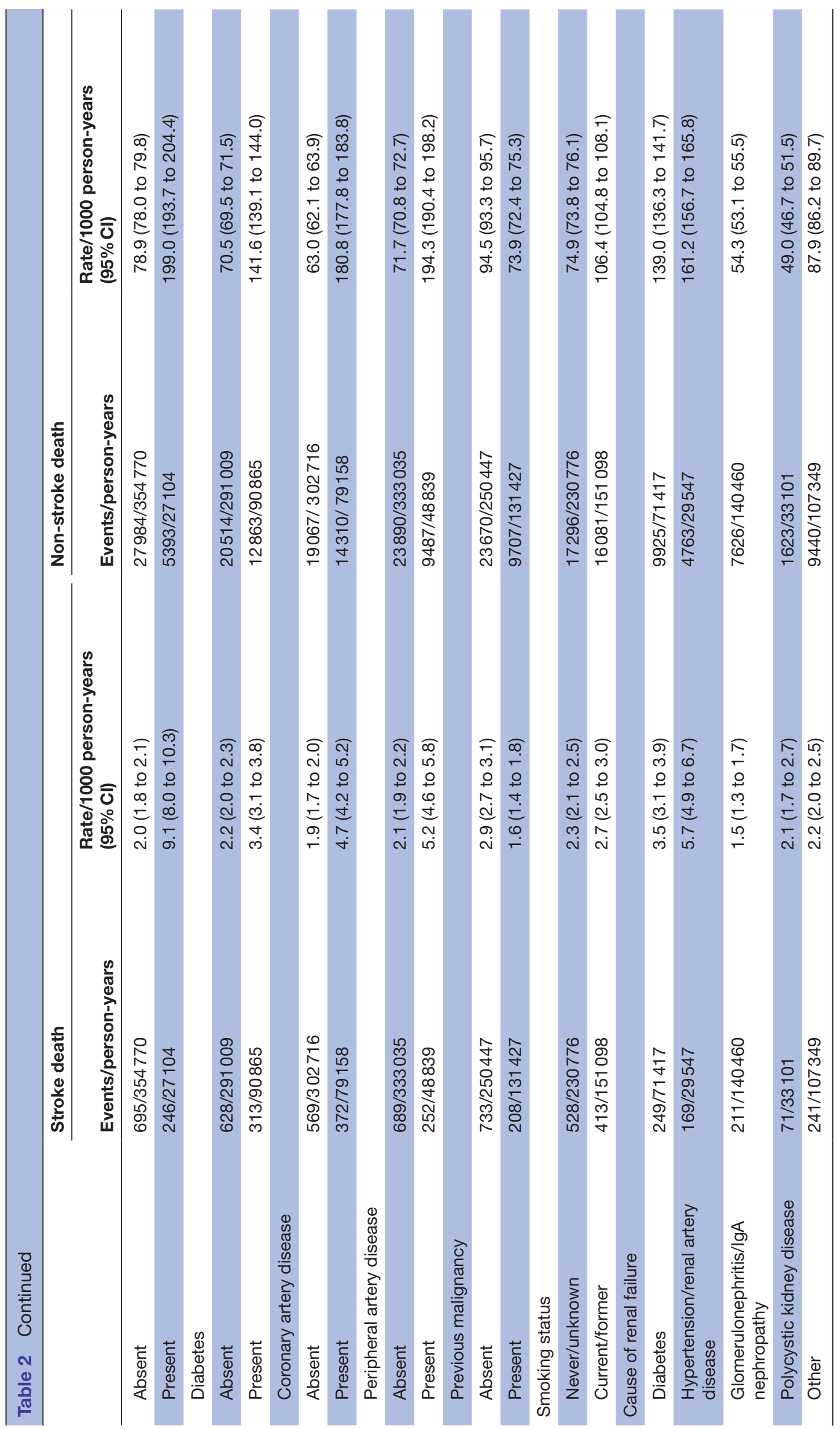


Table 3 Summary of the adjusted sub-HR (SHR) estimates for the competing risks model with stroke deaths as the main event and non-stroke deaths as the competing event

\begin{tabular}{|c|c|c|c|c|}
\hline & \multicolumn{2}{|l|}{ Stroke death } & \multicolumn{2}{|l|}{ Non-stroke death } \\
\hline & SHR (95\% Cl) & $P$ value & SHR (95\% Cl) & $P$ value \\
\hline Age at study entry (years) & & $<0.001$ & & $<0.001$ \\
\hline$\leq 29$ & $0.43(0.26$ to 0.70$)$ & - & 0.39 (0.37 to 0.43$)$ & - \\
\hline $30-49$ & Ref & & Ref & \\
\hline $50-64$ & 1.47 (1.16 to 1.86$)$ & - & 1.96 (1.89 to 2.04$)$ & - \\
\hline $65-74$ & 1.82 (1.42 to 2.33$)$ & - & 3.23 (3.10 to 3.37 ) & - \\
\hline$\geq 75$ & 1.92 (1.45 to 2.55$)$ & - & 4.68 (4.45 to 4.91$)$ & - \\
\hline \multicolumn{5}{|l|}{ Sex } \\
\hline Male & Ref & & Ref & \\
\hline Female & 1.41 (1.21 to 1.64$)$ & $<0.001$ & 1.05 (1.02 to 1.08$)$ & $<0.001$ \\
\hline Year of ESKD & & $<0.001$ & & $<0.001$ \\
\hline$\leq 1995$ & 1.93 (1.56 to 2.39$)$ & - & 1.39 (1.33 to 1.45$)$ & - \\
\hline 1996-2005 & 1.30 (1.09 to 1.55$)$ & - & 1.29 (1.25 to 1.33$)$ & - \\
\hline 2006-2013 & Ref & & Ref & \\
\hline BMI category & & $<0.001$ & & $<0.001$ \\
\hline Underweight & 0.78 (0.54 to 1.12$)$ & - & 1.24 (1.16 to 1.33$)$ & - \\
\hline Normal & Ref & & Ref & \\
\hline Overweight & $0.76(0.64$ to 0.90$)$ & - & 0.93 (0.90 to 0.96$)$ & - \\
\hline Obese & 0.61 (0.49 to 0.76$)$ & - & 0.98 (0.95 to 1.02$)$ & - \\
\hline \multicolumn{5}{|l|}{ Racial background } \\
\hline Non-white & Ref & & Ref & \\
\hline White & 0.88 (0.73 to 1.06$)$ & 0.182 & 1.11 (1.07 to 1.15$)$ & $<0.001$ \\
\hline \multicolumn{5}{|l|}{ Comorbidities at ESKD } \\
\hline \multicolumn{5}{|l|}{ Cerebrovascular disease } \\
\hline Present & 2.39 (1.99 to 2.87$)$ & $<0.001$ & $1.13(1.09$ to 1.17$)$ & $<0.001$ \\
\hline \multicolumn{5}{|l|}{ Coronary artery disease } \\
\hline Present & 1.00 (0.83 to 1.20$)$ & 0.960 & 1.38 (1.34 to 1.42$)$ & $<0.001$ \\
\hline \multicolumn{5}{|l|}{ Peripheral artery disease } \\
\hline Present & 0.90 (0.74 to 1.09$)$ & 0.299 & 1.30 (1.25 to 1.34$)$ & $<0.001$ \\
\hline \multicolumn{5}{|l|}{ Previous malignancy } \\
\hline Present & $0.64(0.53$ to 0.78$)$ & $<0.001$ & 0.90 (0.88 to 0.93$)$ & $<0.001$ \\
\hline \multicolumn{5}{|l|}{ Smoking status } \\
\hline Never/unknown & Ref & & Ref & \\
\hline Current/former & $1.06(0.91$ to 1.24$)$ & 0.441 & 1.18 (1.15 to 1.21$)$ & $<0.001$ \\
\hline First dialysis module & & 0.015 & & $<0.001$ \\
\hline HD & Ref & & Ref & \\
\hline PD & 0.85 (0.72 to 1.01$)$ & - & 1.00 (0.97 to 1.03$)$ & - \\
\hline Transplant & 0.27 (0.09 to 0.84$)$ & - & $0.26(0.22$ to 0.31$)$ & - \\
\hline Cause of renal failure & & 0.020 & & $<0.001$ \\
\hline Diabetes & 1.23 (0.99 to 1.54$)$ & - & 1.60 (1.54 to 1.66$)$ & - \\
\hline $\begin{array}{l}\text { Hypertension/ } \\
\text { renal artery disease }\end{array}$ & 1.39 (1.09 to 1.78$)$ & - & 1.23 (1.18 to 1.29$)$ & - \\
\hline $\begin{array}{l}\text { Glomerulonephritis/ } \\
\text { IgA nephropathy }\end{array}$ & Ref & & Ref & \\
\hline Polycystic kidney disease & $1.38(1.00$ to 1.90$)$ & - & $0.72(0.68$ to 0.76$)$ & - \\
\hline
\end{tabular}




\begin{tabular}{|c|c|c|c|c|}
\hline & \multicolumn{2}{|l|}{ Stroke death } & \multicolumn{2}{|l|}{ Non-stroke death } \\
\hline & SHR (95\% CI) & $P$ value & SHR $(95 \% \mathrm{Cl})$ & $P$ value \\
\hline Other & $1.03(0.82$ to 1.28$)$ & - & $1.42(1.37$ to 1.47$)$ & - \\
\hline
\end{tabular}

The multivariate model was also adjusted for country. Global $p$ values were test for heterogeneity. Bold values indicate estimates with a significant association at 0.05 alpha level (ie, $p$ value $<0.05$ ). $\mathrm{BMI}$, body mass index; ESKD, end-stage kidney disease; HD, haemodialysis; PD, peritoneal dialysis.

polycystic kidney disease were $39 \%$ (SHR $1.50,95 \% \mathrm{CI}$ 1.20 to 1.87 ) and $38 \%$ (SHR $1.38,95 \%$ CI 1.00 to 1.90 ), respectively, more likely of stroke death compared with those with glomerulonephritis/IgA nephropathy. The unadjusted estimated risk of stroke death is presented in online supplementary appendix 3 .

In the sensitivity analysis, the risk of fatal ischaemic stroke was not associated with BMI, cerebrovascular disease, previous malignancy or HD compared with transplant (online supplementary appendix 4). White racial background and coronary artery disease were associated with an increased risk of ischaemic stroke death, which were not risk factors for all-cause stroke death. Year of ESKD was also not associated with the risk of fatal ischaemic or haemorrhagic stroke. The risk factors for fatal haemorrhagic stroke were all similar to the risk factors for all-cause stroke death, except for cause of renal failure (online supplementary appendix 4). Cause of renal failure was not associated with the risk of fatal haemorrhagic stroke.

There were an additional 694 stroke deaths when all-cause stroke death was defined as the underlying cause or secondary cause when kidney failure was the underlying cause. All risk factors associated with the extended definition for all-cause stroke were the same as those identified in the main analysis, except for peripheral artery disease (online supplementary appendix 5). In the sensitivity multivariate model, patients with pre-existing peripheral artery disease were at an increased risk for all-cause stroke mortality.

\section{Risk factors for non-stroke mortality}

The multivariable model suggested that a higher risk of non-stroke death was associated with older age, female sex, earlier year of ESKD, underweight or normal BMI range, Caucasian background, presence of comorbidities including cerebrovascular disease, coronary artery disease, peripheral artery disease and no previous malignancy, current or former smoker, dialysis compared with transplant and cause of ESKD including diabetes, hypertension/renal artery disease or other causes (table 3).

Patients initiating ESKD treatment with a transplant were associated with a $73 \%$ lower risk of non-stroke death compared with those initiating HD (SHR 0.27, 95\% CI 0.23 to $0.32 ; \mathrm{p}<0.001)$. Similar estimates for the risk of non-stroke death were produced when transplant was considered a time-varying covariate (HR $0.30,95 \%$ CI 0.29 to $0.32 ; \mathrm{p}<0.001$ ) (online supplementary appendix
2). The cause of ESKD was also significantly associated with non-stroke mortality $(p<0.001)$. A higher risk of non-stroke mortality was associated with diabetes (SHR $1.60,95 \%$ CI 1.54 to 1.65 ), hypertension/renal artery disease (SHR 1.24, 95\% CI 1.19 to 1.29 ) or other causes (SHR 1.38, 95\% CI 1.33 to 1.42) compared with glomerulonephritis/IgA nephropathy as the cause of ESKD. Those with polycystic kidney disease were $24 \%$ less likely (SHR $0.76,95 \%$ CI 0.72 to 0.80 ) to die of non-stroke compared with those with glomerulonephritis/IgA nephropathy. The unadjusted estimated risk of non-stroke death is presented in online supplementary appendix 3 .

\section{Cumulative incidence of death}

The estimated cumulative incidence of stroke mortality since RRT was relatively low at $0.5 \%$ at 2 years, while the non-stroke mortality was substantially higher at $15.5 \%$ at 2 years. At 5 years, the cumulative incidence increased to $0.9 \%$ for stroke and $36.8 \%$ for non-stroke mortality.

The cumulative incidence of stroke or non-stroke death differed by sex, where females consistently had a higher cumulative incidence (figure 2A). For stroke mortality, the cumulative incidence at 2 years in males was $0.4 \%$ and slightly higher in females at $0.6 \%$. At 5 years, the sex difference increased where the cumulative incidence was $0.8 \%$ in males and $1.1 \%$ in females. For non-stroke mortality, the cumulative incidence at 2 years was similar in males at $17.6 \%$ and females at $17.4 \%$. At 5 years, the cumulative incidence remained similar at $43.3 \%$ in males and $42.9 \%$ in females.

The cumulative incidence of stroke mortality decreased over time, where those diagnosed with ESKD in 20062013 had the lowest cumulative incidence during follow-up (figure 2B). At 5 years, the cumulative incidence increased to $1.6 \%$ in $\leq 1995,0.9 \%$ in $1996-2005$ and $0.7 \%$ in 2006-2013. The cumulative incidence of non-stroke mortality showed a similar trend. At 5 years, the cumulative incidence increased to $46.3 \%$ in $\leq 1995$, $38.0 \%$ in $1996-2005$ and $30.4 \%$ in $2006-2013$.

\section{DISCUSSION}

This multinational study represents the largest study of stroke mortality in people with ESKD to date, including more than 381000 PYs follow-up in 60823 people starting treatment for ESKD to evaluate risk factors associated with stroke mortality in the Australia and New Zealand. Our main finding was that women with ESKD were $41 \%$ 
A
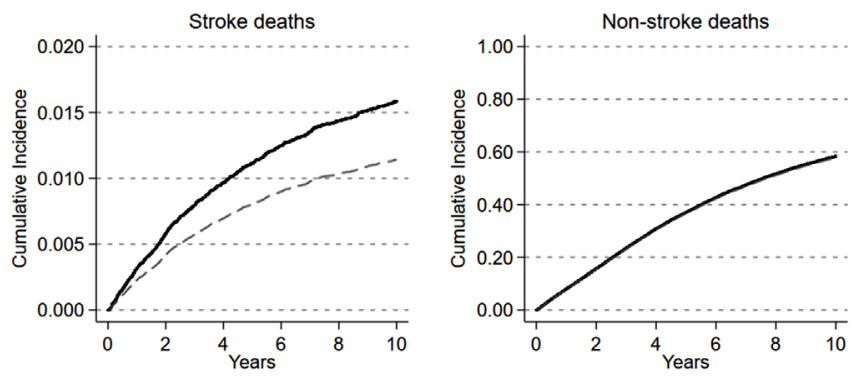

$$
\text { Female }---- \text { Male }
$$

B
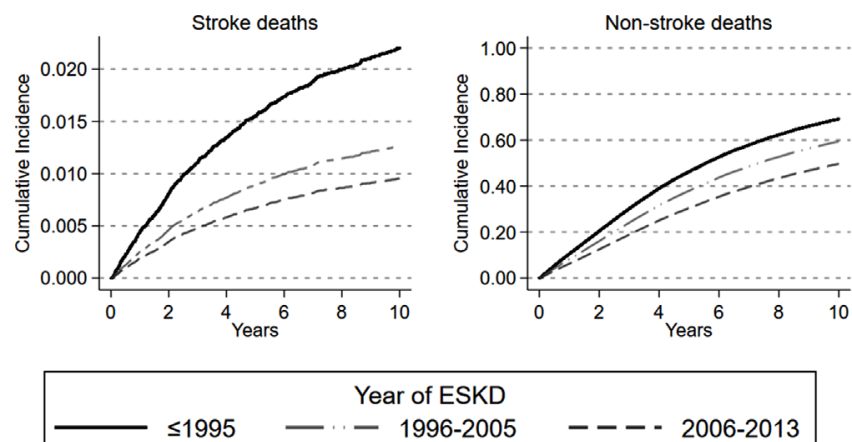

- - - 2006-2013

Figure 2 Estimate cumulative incidence of stroke and nonstroke deaths, by: (A) sex; (B) year period of ESKD diagnosis. ESKD, end-stage kidney disease.

more likely to die from a stroke death compared with men with ESKD. There was a decreasing trend in stroke mortality risk over time, where patients with incident ESKD in $\leq 1995$ nearly twice as likely to die of stroke death compared those starting ESKD treatment in 2006-2013. We also found that those with cerebrovascular disease were over twofold more likely to die of stroke compared with those without cerebrovascular disease. Other factors associated with an increased stroke mortality risk included older age, normal BMI (vs overweight or obese BMI), no previous malignancy, dialysis treatment compared transplant and hypertension/renal artery disease or diabetes as the cause of ESKD (vs glomerulonephritis/ IgA nephropathy). These risk factors highlight patients with ESKD at greater risk of stroke death that could be targeted in prevention strategies, particularly those with cerebrovascular disease and females.

Our findings suggest a sex difference in stroke mortality where women have at a greater risk of dying following a stroke compared with men. Women have also been shown to have a stroke incidence nearly twice that of men in the Australian ESKD population. This is unlike the Australian and New Zealand general population, where men have a higher stroke incidence but comparable death rate to women. ${ }^{20}{ }^{21}$ Women may have confounding factors that place them at a greater risk of stroke death. In the general population, women are older at stroke onset, have poorer outcomes after stroke and are more likely to have atrial fibrillation and hypertension. ${ }^{22-25}$ Alternatively, stroke prevention may not be implemented in women as often as men. Previous studies in the general population have found that women were less likely to be prescribed antiplatelet therapy following an ischaemic stroke and less frequently treated with an oral anticoagulant once diagnosed with atrial fibrillation. ${ }^{2627}$ These gaps in care may also prevail in the ESKD population, however, no studies to date have specifically examined sex differences in stroke prevention among patients with ESKD. Whether a lack of stroke prevention strategies or other factors contribute to female patients with ESKD being at a greater risk of stroke and stroke death still needs further investigation.

Pre-existing cerebrovascular disease presents an opportunity for secondary prevention in the ESKD population, either with existing or novel interventions. Our findings are supported by previous studies of incident and recurrent strokes in the ESKD population. ${ }^{28-31}$ Studies in the UK found that patients with ESKD with prior cerebrovascular disease were 4.5 times more at risk of incident stroke. ${ }^{28} 31$ The mechanisms underlying cerebrovascular disease and ESKD are likely due to a combination of factors. ${ }^{32-35}$ First, CKD and cerebrovascular disease share common risk factors, including older age, diabetes, hypertension, obesity and smoking. ${ }^{33} 34$ Second, CKD-associated risk factors associated with $\mathrm{CKD}$, such as chronic inflammation, oxidative stress and uraemic retention products, contribute to an excess risk of cerebrovascular disease. ${ }^{32} 35$ Third, the dialysis process itself could lead to an increased risk of stroke, such as hypoperfusion in HD and glucose degradation in PD. ${ }^{96}$ In transplant patients, graft function and the effect of albuminuria and eGFR may also modify their risk of stroke death. ${ }^{10}$ Until now cerebrovascular disease prevention strategies implemented in the ESKD population have mainly been based on stroke interventions in the general population. ${ }^{33} 3738$ Secondary stroke prevention can either target modifiable risk factors, such as hypertension and dyslipidaemia, or recommend the use of an anticoagulant or antiplatelet. ${ }^{38}{ }^{39}$ However, the effectiveness of anticoagulant or antiplatelet therapy in the ESKD population is unclear as patients with ESKD are often under-represented or excluded from prevention trials. ${ }^{33}$ The few observational studies in anticoagulation therapy with warfarin suggested little evidence of preventing ischaemic strokes and increased the risk of major bleeding among patients with ESKD undergoing HD. ${ }^{40}$ Similarly, there is an absence of trial data on the safety and effectiveness of stroke thrombolysis in people with ESKD. A study examining nephrologist views on stroke thrombolysis showed that most had concerns about the bleeding risks and were uncertain about the overall benefits in patients with ESKD. ${ }^{42}$ However, our findings did suggest a decreasing trend in stroke mortality risk over time where those initiating ESKD treatment in recent years were less likely to die of stroke. This may have arisen from improved stroke prevention interventions over time or better renal management. Further 
research is still needed to assess whether the effectiveness of secondary stroke prevention could be improved in the ESKD population.

There were similarities and differences in the risk factors associated with stroke and non-stroke mortality. Several risk factors were associated with an increased risk for both stroke and non-stroke death, including older age, earlier year of ESKD, normal BMI compared with overweight BMI, cerebrovascular disease, HD compared with transplant and diabetes or hypertension/renal artery disease compared with glomerulonephritis/IgA nephropathy as cause of renal failure. Female sex was only associated with an increased risk of stroke death. While factors only associated with an increased risk of non-stroke death were underweight BMI compared with normal BMI, white racial background, coronary or peripheral artery disease, current or former smoker and glomerulonephritis/IgA nephropathy compared with polycystic kidney disease as cause of ESKD.

A major strength of our study was our large population-based patient sample that included the entire ESKD population in Australia and New Zealand. We also conducted several sensitivity analyses which were relatively consistent in the risk factors for stroke mortality. This suggests our main analysis was fairly robust and reliable. However, there were several limitations to our study. The limited data collected by ANZDATA did not allow us to evaluate whether secondary stroke prevention interventions were being used in patients with ESKD. In addition, previous studies have shown family history of stroke, blood pressure, atrial fibrillation and laboratory-related data (such as haemoglobin) are strongly associated with stroke, yet these data were not available in our patients with ESKD as it is not systematically collected by the ANZDATA registry. We also used data linkage to establish the cause of death and date of death. Australian patients were matched using probabilistic linkage to the national death registry, while New Zealand patients were matched via deterministic linkage. Probabilistic linkage can lead to patients being incorrectly linked and, subsequently, reporting an incorrect cause of death. The false positive rate is estimated at 5 per 1000 or $0.5 \% .^{43}$ Mismatched fact of death was high at $8 \%$ for Australian patients and $6 \%$ for New Zealand patients. The national death registries would not have captured patient deaths if they had occurred outside of their registered country. However, relatively few patients $(0.8 \%)$ were considered lost to follow-up in ANZDATA, where overseas death would be possible. Hence, it is unlikely that patients with ESKD moving overseas or between Australia and New Zealand would have contributed to reduced linkage quality. In addition, we used the underlying cause of death to define stroke deaths. We would, therefore, expect these stroke deaths to include patients who died of stroke and stroke complications. Any misclassification bias would be expected to be non-differential among people with ESKD and the general population. We used the Fine and Gray model to account for competing events of non-stroke deaths, which does not allow time-varying covariates. We were therefore unable to evaluate treatment modality switches during follow-up. Finally, the sensitivity analyses for risk factors associated with ischaemic stroke deaths and haemorrhagic stroke deaths separately had substantially fewer stroke deaths than the overall main analysis. In particular, there were only 108 ischaemic stroke deaths which reduced the power to detect associated risk factors in the model and increased impression of SHR estimates. As such, we advise caution when interpreting these findings to suggest risk factors for ischaemic stroke deaths are significantly different to haemorrhagic or all-cause stroke deaths in patients with ESKD.

In conclusion, our analysis describes risk factors for stroke mortality in the Australian and New Zealand ESKD population. Most factors that led to an increased risk of stroke death were non-modifiable, including older age, female sex, year of ESKD and cause of ESKD. The sex difference in stroke mortality in the ESKD population is not seen in the general population. This raises questions as to whether female patients with ESKD have other clinical characteristics that place them at a greater risk or whether prevention strategies are not prescribed in women as often as men. In addition, implementing prevention strategies in those with prior cerebrovascular disease has the potential to prevent secondary stroke in patients with ESKD. It is unclear whether stroke prevention strategies are as effective in the ESKD population as in the general population. Further research into the use and effectiveness of stroke prevention in the ESKD population is needed to evaluate means of reducing stroke mortality in those with cerebrovascular disease and in females.

Contributors ACW, PM, MA-R and PJK contributed to the concept development. NLDLM, MA-R and PJK contributed to the statistical design. NLDLM and MA-R performed the statistical analysis. NLDLM and PM prepared the draft manuscript. All authors (ACW, PM, MA-R, PJK and RA-SS) contributed to the interpretation of the results, critically revised the manuscript and approved of the final manuscript for submission.

Funding The data reported here have been supplied by the Australia and New Zealand Dialysis and Transplant Registry (ANZDATA), the Australian Institute of Health and Welfare (AlHW) and the New Zealand Ministry of Health. We would like to acknowledge the assistance provided by the AlHW and New Zealand Ministry of Health in the data linkage process and Kidney Health Australia for their support. The interpretation and reporting of these data are the responsibility of the Editors and in no way should be seen as an official policy of interpretation of ANZDATA, AlHW or the New Zealand.

Competing interests None declared.

Patient consent for publication Not required.

Ethics approval This study received ethics approval from the University of Sydney (Project No.: 2014/917), AlHW (Reference No.: E02015/3/181) and the New Zealand Ministry of Health (Reference No.: 14/NTB/171).

Provenance and peer review Not commissioned; externally peer reviewed.

Data sharing statement De-identified data may be made available by request to any qualified investigator, subject to appropriate ethics and data custodian approvals.

Open access This is an open access article distributed in accordance with the Creative Commons Attribution Non Commercial (CC BY-NC 4.0) license, which permits others to distribute, remix, adapt, build upon this work non-commercially, and license their derivative works on different terms, provided the original work is 
properly cited, appropriate credit is given, any changes made indicated, and the use is non-commercial. See: http://creativecommons.org/licenses/by-nc/4.0/.

\section{REFERENCES}

1. Collins AJ. Cardiovascular mortality in end-stage renal disease. Am J Med Sci 2003;325:163-7.

2. Schmid H, Schiffl H. Hemodiafiltration and survival of end-stage renal disease patients: the long journey goes on. Int Urol Nephrol 2012;44:1435-40.

3. Villar E, Chang SH, McDonald SP. Incidences, treatments, outcomes, and sex effect on survival in patients with end-stage renal disease by diabetes status in Australia and New Zealand (1991 2005). Diabetes Care 2007;30:3070-6.

4. Roberts MA, Polkinghorne KR, McDonald SP, et al. Secular trends in cardiovascular mortality rates of patients receiving dialysis compared with the general population. Am J Kidney Dis 2011;58:64-72.

5. Cherng YG, Lin CS, Shih CC, et al. Stroke risk and outcomes in patients with chronic kidney disease or end-stage renal disease: Two nationwide studies. PLoS One 2018;13:e0191155.

6. Keith DS, Nichols GA, Gullion CM, et al. Longitudinal follow-up and outcomes among a population with chronic kidney disease in a large managed care organization. Arch Intern Med 2004;164:659-63.

7. Longenecker JC, Coresh J, Powe NR, et al. Traditional cardiovascular disease risk factors in dialysis patients compared with the general population: the CHOICE Study. J Am Soc Nephrol 2002;13:1918-27.

8. Seliger SL, Gillen DL, Tirschwell D, et al. Risk factors for incident stroke among patients with end-stage renal disease. J Am Soc Nephrol 2003;14:2623-31.

9. Grantham CE, Hull KL, Graham-Brown MPM, et al. The Potential Cardiovascular Benefits of Low-Glucose Degradation Product, Biocompatible Peritoneal Dialysis Fluids: A Review of the Literature. Perit Dial Int 2017;37:375-83.

10. Lentine KL, Rocca Rey LA, Kolli S, et al. Variations in the risk for cerebrovascular events after kidney transplant compared with experience on the waiting list and after graft failure. Clin J Am Soc Nephrol 2008;3:1090-101.

11. Australian Bureau of Statistics. General Community Profile. Adelaide Australia. 2016 http://www.censusdata.abs.gov.au/census_services/ getproduct/census/2016/communityprofile/036?opendocument (Cited 13 Feb 2018)

12. Statistics New Zealand. New Zealand in Profile 2015: An overview of New Zealand's people, economy and environment. Wellington, New Zealand, 2015. http://archive.stats.govt.nz/browse_for_stats/ snapshots-of-nz/nz-in-profile-2015.aspx. (Cited 13 Feb 2018).

13. McDonald SP, Russ GR. Australian registries-ANZDATA and ANZOD. Transplant Rev 2013;27:46-9.

14. Health, N.C.f.C.i. The International Statistical Classification of Diseases and Related Health Problems, 10th Revision, Australian modification. 9th ed, 2015.

15. Australian Institute of Health and Welfare. Stroke and its management in Australia: an update. Canberra: Australian Institute of Health and Welfare, 2013. https://www.aihw.gov.au/getmedia/3d56c949-68a446f3-bc7c-c40c89904d38/13994.pdf.aspx?inline=true. (Cited 23 Apr 2018)

16. Kokotailo RA, Hill MD. Coding of stroke and stroke risk factors using international classification of diseases, revisions 9 and 10. Stroke 2005;36:1776-81.

17. Becker R, Silvi J, Ma Fat D, et al. A method for deriving leading causes of death. Bull World Health Organ 2006;84:297-304.

18. Fine JP, Gray RJ. A proportional hazards model for the subdistribution of a competing risk. J Am Stat Assoc 1999;94:496-509.

19. Blakely T, Salmond C. Probabilistic record linkage and a method to calculate the positive predictive value. Int J Epidemiol 2002;31:1246-52.

20. Barker-Collo S, Bennett DA, Krishnamurthi RV, et al. Sex differences in stroke incidence, prevalence, mortality and disability-adjusted life years: Results from the global burden of disease study 2013. Neuroepidemiology 2015;45:203-14.

21. Reeves MJ, Bushnell CD, Howard G, et al. Sex differences in stroke: epidemiology, clinical presentation, medical care, and outcomes. Lancet Neurol 2008;7:915-26.

22. Di Carlo A, Lamassa M, Baldereschi M, et al. Sex differences in the clinical presentation, resource use, and 3-month outcome of acute stroke in Europe: data from a multicenter multinational hospitalbased registry. Stroke 2003;34:1114-9.

23. Holroyd-Leduc JM, Kapral MK, Austin PC, et al. Sex differences and similarities in the management and outcome of stroke patients. Stroke 2000;31:1833-7.

24. Kapral MK, Fang J, Hill MD, et al. Sex differences in stroke care and outcomes: results from the Registry of the Canadian Stroke Network. Stroke 2005;36:809-14.

25. Roquer J, Campello AR, Gomis M. Sex differences in first-ever acute stroke. Stroke 2003;34:1581-5.

26. Glader EL, Stegmayr B, Norrving B, et al. Sex differences in management and outcome after stroke: a Swedish national perspective. Stroke 2003;34:1970-5.

27. Simpson CR, Wilson C, Hannaford PC, et al. Evidence for age and sex differences in the secondary prevention of stroke in Scottish primary care. Stroke 2005;36:1771-5.

28. Findlay MD, Thomson PC, Fulton RL, et al. Risk factors of ischemic stroke and subsequent outcome in patients receiving hemodialysis. Stroke 2015;46:2477-81.

29. Masson P, Kotwal S, Kelly PJ, et al. Risk factors for stroke in people with end-stage kidney disease: A cohort study. Cerebrovasc Dis 2016;42(5-6):428-38.

30. Sozio SM, Armstrong PA, Coresh J, et al. Cerebrovascular disease incidence, characteristics, and outcomes in patients initiating dialysis: the choices for healthy outcomes in caring for ESRD (CHOICE) study. Am J Kidney Dis 2009;54:468-77.

31. Power A, Chan K, Singh SK, et al. Appraising stroke risk in maintenance hemodialysis patients: a large single-center cohort study. Am J Kidney Dis 2012;59:249-57.

32. El Husseini N, Kaskar O, Goldstein LB. Chronic kidney disease and stroke. Adv Chronic Kidney Dis 2014;21:500-8.

33. Herrington W, Haynes R, Staplin N, et al. Evidence for the prevention and treatment of stroke in dialysis patients. Semin Dial 2015;28:35-47.

34. Saeed F, Kousar N, Qureshi K, et al. A review of risk factors for stroke in patients with chronic kidney disease. J Vasc Interv Neurol 2009;2:126-31.

35. Toyoda K, Ninomiya T. Stroke and cerebrovascular diseases in patients with chronic kidney disease. Lancet Neurol 2014;13:823-33.

36. Prohovnik I, Post J, Uribarri J, et al. Cerebrovascular effects of hemodialysis in chronic kidney disease. J Cereb Blood Flow Metab 2007;27:1861-9.

37. Khella S, Bleicher MB. Stroke and its prevention in chronic kidney disease. Clin J Am Soc Nephrol 2007;2:1343-51.

38. Khella SL. New insights into stroke in chronic kidney disease. Adv Chronic Kidney Dis 2008;15:338-46.

39. Townsend RR. Stroke in chronic kidney disease: prevention and management. Clin J Am Soc Nephrol 2008;3 Suppl 1(Suppl 1):S11-S16.

40. Dahal K, Kunwar S, Rijal J, et al. Stroke, major bleeding, and mortality outcomes in warfarin users with atrial fibrillation and chronic kidney disease: A meta-analysis of observational studies. Chest 2016;149:951-9.

41. Winkelmayer WC, Liu J, Setoguchi S, et al. Effectiveness and safety of warfarin initiation in older hemodialysis patients with incident atrial fibrillation. Clin J Am Soc Nephrol 2011;6:2662-8.

42. Power A, Fogarty D, Wheeler DC. Acute stroke thrombolysis in end-stage renal disease: a national survey of nephrologist opinion. Nephron Clin Pract 2013;124(3-4):167-72.

43. Centre for Health Record Linkage. Master Linkage Key Quality Assurance. Sydney, Australia, 2012. http://www.cherel.org.au/media/ 24160/qa_report_2012-a.pdf. (Cited 13 Mar 2018). 